\title{
A test of statistical hadronization with exclusive rates in $\mathrm{p} \overline{\mathrm{p}}$ annihilation at rest
}

\author{
Lorenzo Ferroni* \\ University of Florence \\ E-mail: ferroniafi.infn.it

\section{Francesco Becattini} \\ University of Florence \& INFN \\ E-mail: becattiniefi.infn.it
}

\begin{abstract}
We calculated the rate of exclusive multihadronic channels in the statistical hadronization model within a full microcanonical framework by enforcing the maximal set of conservation laws including energy-momentum, angular momentum, parity, as well as isospin, C-parity and charges. A preliminary test has been performed by comparing model predictions against a set of two-mesons channel rates measured in $\mathrm{p} \overline{\mathrm{p}}$ annihilation at rest. The same comparison has been made for the phase space dominance model. The analysis suffers from the complication of the annihilation process which proceeds from many initial $\mathrm{p} \overline{\mathrm{p}}$ atomic bound states, thus requiring the introduction of several free parameters. Both models prove to reproduce the general trend of the experimental data although the agreement with the specific values is not satisfactory.
\end{abstract}

Critical Point and Onset of Deconfinement 4th International Workshop

July 9-132007

GSI Darmstadt,Germany

${ }^{*}$ Speaker. 


\section{Introduction}

In the last three decades, many analyses confirmed that the statistical hadronization model (SHM) successfully reproduces particle abundances and transverse momentum spectra in high energy collisions $(\sqrt{s} \gtrsim 10 \mathrm{GeV})$ of elementary particles and heavy ions [1,2]. These results triggered a debate about the interpretation of the model $[3,4,5,6,7]$. In fact, the fundamental assumption of statistical equilibrium, cannot be explained, at least in elementary particles collisions, in terms of a collisional processes at the level of formed hadrons because the system expands too quickly, and also in heavy ions collisions kinetic calculations seems to confirm this fact [6]. Therefore, the apparent statistical equilibrium, must be an inherent property of the hadronization process itself (as was pointed out by Hagedorn many years ago [8]), that is: hadrons are born at equilibrium.

Apart from a genuine statistical equilibrium within a finite volume, another scenario has been proposed to account for this peculiar feature of hadron production: the so-called phase space dominance [5]. According to this model, the apparent statistical equilibrium is the result of a special property of the dynamical matrix element governing hadron emission from pre-hadronic massive clusters, which weakly depends on final state momenta and particle species. Thereby, a statisticallike emission ensues [7] which has nothing to do with a properly defined statistical system, i.e. there is no finite volume and neither entropy nor temperature can be introduced.

As has been discussed in detail in [7] (and briefly summarized in the following), both genuine statistical equilibrium and phase space dominance are highly non-trivial hypotheses, and before discussing possible mechanisms responsible for the apparent equilibrium features it would be at least desirable to discriminate between the two aforementioned scenarios.

Another fascinating explanation of the observed statistical equilibrium has been recently put forward in refs. [4, 9] where the authors argue an analogy between hadron emission and the UnruhHawking effect. However, the discussion of the latter hypothesis goes beyond our scopes.

The aim of this work is to describe a more stringent test of genuine statistical equilibrium. For this purpose, we have analyzed the production rates of exclusive channels, which have been proposed in [7] as a more effective probe with respect to inclusive hadron multiplicities. In fact, being far less inclusive quantities, exclusive rates could be sensitive enough to finite-volume and dynamical effects to allow drawing some conclusion.

Exclusive rates measurements are available at energies significantly below $10 \mathrm{GeV}$. In calculating model predictions in this scenario, none of the relevant conservation laws, including energymomentum, angular momentum, parity and isospin can be neglected, as pointed out in ref. [10]. Therefore, in a statistical mechanics language, one must calculate the most general microcanonical ensemble where all these quantities are properly conserved.

In two recent publications [11, 12], the microcanonical partition function has been calculated in a field theory framework (in order to account for small-volume effects) enforcing the conservation of the maximal set of observables pertaining to space-time symmetries (the orthochronous Poincaré group): energy-momentum, spin, helicity, parity.

Taking advantage of the formalism developed therein, we calculated the probability of exclusive channels also enforcing the conservation of the internal quantities conserved by strong interaction (isospin, C-parity and abelian charges). We then made a preliminary test on $\mathrm{p} \overline{\mathrm{p}}$ annihilation 
at rest also comparing our results with the corresponding predictions of the phase space dominance model.

\section{Statistical hadronization and phase space dominance}

In the modern formulation of the statistical hadronization model, as a consequence of a collision, a set of extended massive objects (called clusters or fireballs) is formed. Each cluster decay into hadrons in a purely statistical fashion, that is, any multi-hadronic state within the cluster compatible with its quantum numbers is equally likely. The collection of all state within the cluster defines the microcanonical ensemble of the cluster itself, which is the best suited framework for evaluating observable quantities as statistical averages. Nevertheless, because of difficulties arising in microcanonical calculations, a comparison with the data as been mostly made as yet by introducing simplifying assumptions in the canonical (or grand-canonical) ensemble, which is far easier to handle, thereby introducing temperature through a saddle-point expansion [13, 14]. In the case of hadron gas, this is possible at relatively low values of masses and volumes $[14,15]$, around 8 $\mathrm{GeV}$ and $20 \mathrm{fm}^{3}$.

Apart from the genuine statistical equilibrium (and from explanations based on other physical models), the main option arising to account for these observations is the phase space dominance model. Its fundamental idea is the similarity between the (classical) phase space volume of a set of particles, or channel, $\left\{N_{j}\right\} \equiv N_{1}, \ldots, N_{k}$ (where $N_{j}$ stands for the multiplicity of the species $j$ ) and the general expression of the decay rate of a massive particle (cluster) in relativistic quantum mechanics.

If we let $P$ be the initial four-momentum, $V$ the volume and let $p_{n} \equiv\left(\varepsilon_{n}, \mathbf{p}_{n}\right)$ be the four momentum of the particle $n$; the phase space volume $\Omega_{\left\{N_{j}\right\}}$ of the channel $\left\{N_{j}\right\}$ turns out to be [13] (in Boltzmann statistics):

$$
\Omega_{\left\{N_{j}\right\}}=\frac{V^{N}}{(2 \pi)^{3 N}}\left\{\prod_{j} \frac{1}{N_{j} !}\left[\int \mathrm{d}^{3} \mathrm{p}\right]^{N_{j}}\right\} \delta^{4}\left(P-\sum_{n} p_{n}\right)
$$

where $N=\sum_{j} N_{j}$. This quantity is proportional to the probability of observing the channel $\left\{N_{j}\right\}$ as a consequence of the decay of a cluster of volume $V$ and momentum $P$.

On the other hand, the expression of the decay rate into the channel $\left\{N_{j}\right\}$ of a massive particle in relativistic quantum mechanics reads:

$$
\Gamma_{\left\{N_{j}\right\}}=\frac{1}{(2 \pi)^{3 N}}\left\{\prod_{j} \frac{1}{N_{j} !}\left[\int \frac{\mathrm{d}^{3} \mathrm{p}}{2 \varepsilon_{j}}\right]^{N_{j}}\right\} \delta^{4}\left(P-\sum_{n} p_{n}\right)\left|M_{f i}\right|^{2}
$$

where $M_{f i}$ is the Lorentz-invariant dynamical matrix element governing the decay. Assuming, for sake of simplicity, spinless particles, $\left|M_{f i}\right|^{2}$ may in principle depend on all relativistic invariants formed out of the four-momenta of the $N$ particles, as well as on all possible isoscalars formed out of the isovector operators. Nevertheless, if we assume $M_{f i}$ to be weakly dependent on kinematical variables, expression (2.2) becomes quite similar to (2.1) were not for the invariant measure (the so-called invariant momentum space $\mathrm{d}^{3} \mathrm{p} / 2 \varepsilon$ instead of the proper phase space $V \mathrm{~d}^{3} \mathrm{p}$ ) and for the absence of any parameter connected to spacial extension. 
This phenomenon is called phase space dominance because the decay rate is governed by the available phase space volume rather than dynamical matrix element. For instance, if we assume that $\left|M_{f i}\right|^{2}=\alpha^{N}$, the whole dynamics reduces to the same multiplicative constant $\alpha$ for each particle in the channel, it can be then shown that the expression of the mean number of particles of the species $j$ is well approximated, at large $N$, by:

$$
\langle n\rangle_{j} \simeq \frac{\alpha}{(2 \pi)^{3}} \int \frac{\mathrm{d}^{3} \mathrm{p}}{2 \varepsilon_{j}} \mathrm{e}^{-\beta \varepsilon_{j}}
$$

which is very similar to a thermal distribution which one obtains from (2.1) at large multiplicities:

$$
\langle n\rangle_{j} \simeq \frac{V}{(2 \pi)^{3}} \int \mathrm{d}^{3} \mathrm{pe}^{-\beta \varepsilon_{j}}
$$

where $\beta=1 / T$ is the inverse temperature. Conversely the constant $\beta$ in Eq. (2.3) is not a temperature, rather a parameter which is related to the hadronization scale. Yet, the ratios of average multiplicities of particles of different species mimic a thermodynamic behavior. The authors of ref. [5] work out a more specific example based on QED and they conclude, quite reasonably, that a fairly good fit to particle multiplicities may be obtained if integral expressions like (2.3) are used instead of an actual Boltzmann integral.

It should be emphasized that phase space dominance is a highly non-trivial assumption. In fact, the recovery of a thermal-like expression like (2.3) owes to a very special form of the matrix element $\left|M_{f i}\right|^{2}$, where both the dependence on kinematical and isospin invariants was disregarded. If a different form, still perfectly legitimate and possible, is assumed, the thermal-like behavior is spoiled. Therefore, an observed phase space dominance in multihadron production is not a trivial fact and tells us something important about the characteristics of non perturbative QCD dynamics, besides providing us with an empirically good model.

We conclude that a deeper test of the model is needed in order to identify a genuine statisticalthermal behavior and distinguish between it and possible pseudo-statistical models like phase space dominance. Indeed, the study of average inclusive multiplicities or inclusive $p_{T}$ spectra does not allow to draw clearcut conclusions because these observables are not sensitive enough to different integration measures (i.e. $V \mathrm{~d}^{3} \mathrm{p}$ versus $\mathrm{d}^{3} \mathrm{p} / 2 \varepsilon$ respectively in (2.4) and (2.3)) and much information is integrated away. We will then study the production rates of exclusive channels, that is the relative probability of observing a well defined set of particles (channel) in the final state of a collision. Thereby, we would compare directly with the experimental data expressions like (2.1) and (2.2) which are more sensitive to the integration measure in the momentum integrals and the shape of dynamical matrix element.

\section{Probability of exclusive channels}

A nice feature of low-energy exclusive-channels data is that, to a very good approximation, all collision energy is spent into particle production. This means that one can assume the formation of a single cluster at rest in the centre-of-mass frame (see the pictorial sketch in fig. (1)), in clear contrast with the physical picture of hadronization at high energy, where the production of multiple clusters proceeding from perturbative parton showers occurs. The big advantage of having a single cluster is 
that the initial kinematical state is completely known, being the cluster's mass and internal quantum numbers fixed by $\sqrt{s}$ and the initial state conditions. On the other hand, the relatively small volume

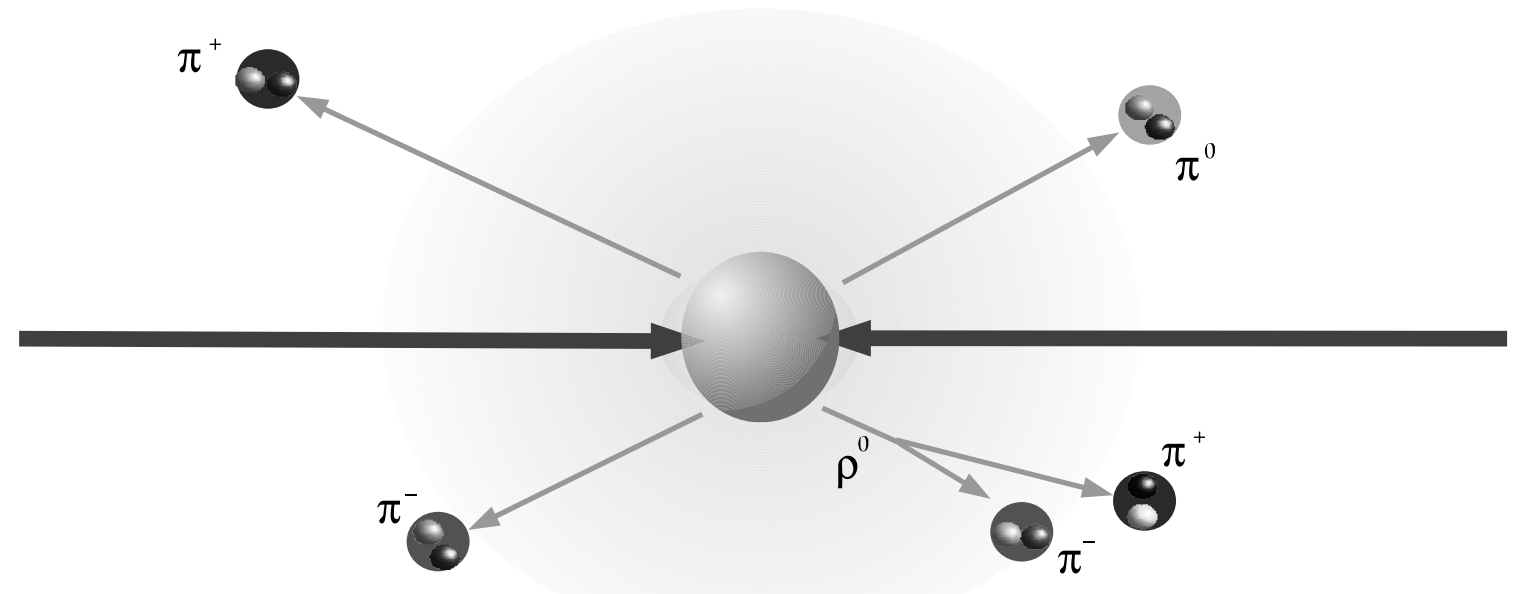

Figure 1: Pictorial representation of a low energy $(\lesssim 5 \mathrm{GeV})$ collision in the statistical model. One single cluster is formed at rest with a mass $M=\sqrt{s}$ and with quantum numbers given by the initial state conditions.

$\left(\mathscr{O}(10) \mathrm{fm}^{3}\right)$ of the cluster, entails some difficulties in the definition of the probability of a channel, or a general final state, with respect to the high-energy case. In fact, the basic postulate of the SHM tells us that localized states compatible with cluster's quantum numbers are equiprobable, but these states do not coincide with observable free-particle asymptotic states. Such difference is, for practical purposes, not an issue when the volume is sufficiently large, but it is relevant in principle and may result in quantitative differences when the volume is comparable with the pion Compton wavelength.

The SHM assumes that the cluster can be described as a normal statistical mixture of multihadronic states $\left|h_{V}\right\rangle$ compatible with its initial quantum numbers. Accordingly, we can write down a microcanonical partition function $\Omega$ [7]:

$$
\Omega=\sum_{h_{V}}\left\langle h_{V}\left|\mathrm{P}_{i}\right| h_{V}\right\rangle
$$

$\mathrm{P}_{i}$ being the projector on the initial state of the cluster:

$$
\mathrm{P}_{i}=\mathrm{P}_{P J \lambda \Pi} \mathrm{P}_{I I_{3}} \mathrm{P}_{C} \mathrm{P}_{\mathbf{Q}}
$$

where $I$ and $I_{3}$ are the isospin and its third component, $C$ is the C-parity ${ }^{1}, \mathbf{Q}=\left(Q_{1}, \ldots, Q_{M}\right)$ a set of $M$ abelian charges (in fact they are 2, baryonic number and strangeness) and where $P_{P J \lambda \Pi}$ is the projector over the maximal set of space-time observables, i.e. an irreducible state of the orthochronous Poincaré group: four-momentum $P$; spin $J$ and its third component $\lambda$; parity $\Pi$. The

\footnotetext{
${ }^{1}$ Of course, the projection $\mathrm{P}_{C}$ makes sense only if $I_{3}=0$ and $\mathbf{Q}=\mathbf{0}$; in this case, $\mathrm{P}_{C}$ commutes with all other projectors
} 
internal symmetry group we are considering in this work is the isospin group $\mathrm{SU}(2)$ and $\mathrm{U}(1)$ 's related to baryonic number and strangeness, thus, we assume $\mathrm{SU}(3)$ flavour symmetry to be completely broken, i.e. $\mathrm{SU}(3) \rightarrow \mathrm{SU}(2) \otimes \mathrm{U}(1)$.

It must be emphasized, that the states $\left|h_{V}\right\rangle$ in (3.1) are not the observable asymptotic free states $|f\rangle$ of the Fock space. Instead, a suitable probability definition should involve these states. To do this, one can first recast the microcanonical partition function (3.1) by using the completeness of states $|f\rangle$ :

$$
\begin{aligned}
\Omega & =\sum_{h_{V}}\left\langle h_{V}\left|\sum_{f}\right| f\right\rangle\left\langle f\left|\mathrm{P}_{i}\right| h_{V}\right\rangle \\
& =\sum_{f}\left\langle f\left|\mathrm{P}_{i} \sum_{h_{V}}\right| h_{V}\right\rangle\left\langle h_{V} \mid f\right\rangle \equiv \sum_{f}\left\langle f\left|\mathrm{P}_{i} \mathrm{P}_{V}\right| f\right\rangle
\end{aligned}
$$

where $\mathrm{P}_{V} \equiv \sum_{h_{V}}\left|h_{V}\right\rangle\left\langle h_{V}\right|$ is the projector onto localized states. We note that the last expression of $\Omega$ in Eq. (3.3) is a proper trace, whereas it was not in Eq. (3.1) as the states $\left|h_{V}\right\rangle$ do not form a complete set of the full Hilbert space.

In [16] (and also in a forthcoming publication [17]), it has been shown that, for a cluster at rest and spherical in shape, a good definition of the probability (for the ideal gas) is:

$$
\rho_{f}=\frac{\left\langle f\left|\mathrm{P}_{i} \mathrm{P}_{V}\right| f\right\rangle}{\Omega} .
$$

According to Eq. (3.3), the microcanonical partition function can be then expressed as a sum over all possible free multiparticle states $|f\rangle$ of the state weight $\omega_{f}$ :

$$
\Omega \equiv \sum_{f} \omega_{f} \quad \text { where } \quad \omega_{f} \equiv\left\langle f\left|\mathrm{P}_{i} \mathrm{P}_{V}\right| f\right\rangle
$$

is proportional to the probability of an asymptotic state $|f\rangle$ :

$$
\rho_{f}=\frac{\omega_{f}}{\Omega}
$$

In turn, the microcanonical partition function can be calculated as an expansion over all possible channels:

$$
\Omega=\sum_{\left\{N_{j}\right\}} \Omega_{\left\{N_{j}\right\}}
$$

The quantity $\Omega_{\left\{N_{j}\right\}}$ is defined as the microcanonical channel weight and it is obtained integrating the state weight $\omega_{f}$ on kinematical variables. In formula, writing a multiparticle state as $|f\rangle \equiv$ $\left|\left\{N_{j}\right\},\{p\}\right\rangle$ where $\{p\}$ stands for set of kinematical variables (momenta and helicities) of particles in the channel, we have:

$$
\Omega_{\left\{N_{j}\right\}}=\sum_{\{p\}} \omega_{f}=\sum_{\{p\}}\left\langle\left\{N_{j}\right\},\{p\}\left|\mathrm{P}_{i} \mathrm{P}_{V}\right|\left\{N_{j}\right\},\{p\}\right\rangle .
$$

The channel weight $\Omega_{\left\{N_{j}\right\}}$ is proportional to the decay probability in the channel $\left\{N_{j}\right\}$ of a cluster with volume $V$ and internal quantum numbers defined by $\mathrm{P}_{i} . \Omega_{\left\{N_{j}\right\}}$ has been calculated in ref. [12] for the ideal relativistic gas, enforcing the maximal set of space-time symmetries, i.e. for 
the projector $\mathrm{P}_{i} \equiv \mathrm{P}_{P J \lambda \Pi}$, that is the projector on an irreducible state of the orthochronous Poincare group $\mathrm{IO}(1,3)^{\uparrow}$.

The projector $\mathrm{P}_{V}$, has been studied in detail in ref. [11] where it has been defined in a quantum field theory framework in order to properly take into account relativistic effects due to the finite volume. In fact, for systems whose linear extension is comparable to the Compton wavelength of involved particles, a fixed number particle description of confined states is not appropriate. One must rather identify localized states as states $|\phi\rangle$ of the quantum fields associated to particles within the system region. For instance, in case of only one scalar particle:

$$
\mathrm{P}_{V}=\int_{V} \mathscr{D} \phi|\phi\rangle\langle\phi|
$$

where $|\phi\rangle \equiv \otimes_{\mathbf{x}}|\phi(\mathbf{x})\rangle$ and $\mathscr{D} \phi$ is the functional measure; the functional integration must be performed getting rid of the degrees of freedom outside the system region $V$ [11]. A generalization of Eq. (3.9) to particles endowed with spin has been provided in ref. [12].

As discussed in $[12,16]$, the state weight of a cluster which is spherically symmetric in shape, does not depend on its polarization $\lambda$. Since we will assume the cluster to be a sharp sphere, we will use a state weight which has been summed over $\lambda$ and divided by $(2 J+1)$. Finally, if we let $N$ be the total number of particles in the channel, i.e. $\sum_{j=1}^{k} N_{j}=N ; S_{j}$ and $\eta_{j}$ respectively the spin and the intrinsic parity of the $j$-th particle species, $p_{n}$ the four-momentum of the $\mathrm{n}$-th particle, the final expression for a cluster at rest is [12]:

$$
\begin{aligned}
& \Omega_{\left\{N_{j}\right\}}=\sum_{\rho}\left[\prod_{j=1}^{k} \frac{\chi\left(\rho_{j}\right)^{b_{j}}}{N_{j} !}\right] \frac{1}{4 \pi} \int_{0}^{4 \pi} \mathrm{d} \psi\left[\prod_{j=1}^{k} \prod_{n_{j}=1}^{N_{j}} \int \mathrm{d}^{3} \mathrm{p}_{n_{j}}\right] \\
\times & \delta^{4}\left(P-\sum_{n=1}^{N} p_{n}\right) \sin \frac{\psi}{2} \sin \left[\left(J+\frac{1}{2}\right) \psi\right] \prod_{j=1}^{k}\left[\prod_{n_{j}=1}^{N_{j}}\left[\frac{\sin \left[\left(S_{j}+\frac{1}{2}\right) n_{j} \psi\right]}{\sin \left(\frac{n_{j} \psi}{2}\right)}\right]^{h_{n_{j}}\left(\rho_{j}\right)}\right] \\
\times & \left(\prod_{j=1}^{k} \prod_{n_{j}=1}^{N_{j}} F_{V}^{(s)}\left(\mathbf{p}_{\rho_{j}\left(n_{j}\right)}-\mathrm{R}_{3}^{-1}(\psi) \mathbf{p}_{n_{j}}\right)+\Pi \prod_{f} \prod_{j=1}^{k} \prod_{n_{j}=1}^{N_{j}} F_{V}^{(s)}\left(\mathbf{p}_{\rho_{j}\left(n_{j}\right)}+\mathrm{R}_{3}^{-1}(\psi) \mathbf{p}_{n_{j}}\right)\right)
\end{aligned}
$$

where

$$
\Pi_{f}=\prod_{j=1}^{k} \eta_{j}^{N_{j}}
$$

and $\boldsymbol{\rho}=\left(\rho_{1}, \ldots, \rho_{k}\right)$ is a set of permutations, $\rho_{j}$ belonging to the permutation group $S_{N_{j}} ; \chi\left(\rho_{j}\right)$ is the parity of the $j$-th permutation and $b_{j}=0,1$ if the species $j$ is a boson or a fermion respectively; the symbol $h_{n_{j}}\left(\rho_{j}\right)$ in (3.10) stands for the number of cyclic permutation with $n_{j}$ elements in $\rho_{j}$ so that $\sum_{n_{j}=1}^{\infty} n_{j} h_{n_{j}}\left(\rho_{j}\right)=N_{j}{ }^{2}$. In Eq. (3.10), $F_{V}^{(s)}$,s are Fourier integrals over a spherically symmetric volume, and for a sharp sphere they read:

$$
\begin{aligned}
& F_{V}^{(\circ)}\left(\mathbf{p}_{\rho(n)}-\mathrm{R}_{3}^{-1}(\psi) \mathbf{p}_{n}\right)=\frac{1}{(2 \pi)^{3}} \int_{V} \mathrm{~d}^{3} \mathrm{xe}^{i \mathbf{x} \cdot\left(\mathbf{p}_{\rho(n)}-\mathrm{R}_{3}^{-1}(\psi) \mathbf{p}_{n}\right)} \\
& =\frac{R^{2}}{2 \pi^{2}} \frac{j_{1}\left(\left|\mathbf{p}_{\rho(n)}-\mathrm{R}_{3}^{-1}(\psi) \mathbf{p}_{n}\right| R\right)}{\left|\mathbf{p}_{\rho(n)}-\mathrm{R}_{3}^{-1}(\psi) \mathbf{p}_{n}\right|}
\end{aligned}
$$

\footnotetext{
${ }^{2}$ The set of integers $h_{1}, \ldots, h_{N} \equiv\left\{h_{n}\right\}$, is usually defined as a partition of the integer $N$ in the multiplicity representation.
} 
$R$ being the radius, $j_{1}$ the spherical Bessel function of the first kind and $\mathrm{R}_{3}(\psi)$ a rotation of an angle $\psi$ along the $z$ axis.

We will give here the generalization of (3.10) where also internal quantum numbers are fully conserved. The calculation has been made in [16] and will be shown in detail in a forthcoming paper [17].

In order to write the general expression of the channel microcanonical weight one needs to first introduce the concept of type and species of particles: particles species differ by whatever quantum number whereas particles belong to the same type if they are light-flavoured mesons belonging to the same isospin multiplet or if they are particle-antiparticle pair. Thus, if $N$ is the total number of particles in a channel $\left\{N_{j}\right\}=\left(N_{1}, \ldots, N_{K}\right)$, we have:

$$
N=\sum_{j=1}^{k} N_{j}=\sum_{j=1}^{K} L_{j}
$$

$N_{j}$ being the multiplicity of species $j$ and $L_{j}$ the multiplicity of the type $j ; k$ be the total number of species and $K$ be total number of types.

If $\chi_{C}$ denotes the product of all intrinsic C-parities ${ }^{3}$ of particles in the channel, the microcanonical channel weight reads:

$$
\begin{aligned}
& \Omega_{\left\{N_{j}\right\}}=\sum_{\left\{\rho_{j}\right\}}\left[\prod_{j=1}^{K} \chi\left(\rho_{j}\right)^{b_{j}}\right] \frac{1}{8 \pi} \int_{0}^{4 \pi} \mathrm{d} \psi\left[\prod_{j=1}^{k} \frac{1}{N_{j} !} \prod_{n_{j}=1}^{N_{j}} \int \mathrm{d}^{3} \mathrm{p}_{n_{j}}\right] \\
& \times \delta^{4}\left(P-\sum_{n=1}^{N} p_{n}\right) \sin \frac{\psi}{2} \sin \left[\left(J+\frac{1}{2}\right) \psi\right] \prod_{j=1}^{K}\left[\prod_{l_{j}=1}^{L_{j}}\left[\frac{\sin \left[\left(S_{j}+\frac{1}{2}\right) l_{j} \psi\right]}{\sin \left(\frac{l_{j} \psi}{2}\right)}\right]^{h_{l_{j}}\left(\rho_{j}\right)}\right] \\
& \times\left(\prod_{j=1}^{K} \prod_{l_{j}=1}^{L_{j}} F_{V}^{(\circ)}\left(\mathbf{p}_{\rho_{j}\left(l_{j}\right)}-\mathrm{R}_{3}^{-1}(\psi) \mathbf{p}_{l_{j}}\right)+\Pi \Pi^{f} \prod_{j=1}^{K} \prod_{l_{j}=1}^{L_{j}} F_{V}^{(\circ)}\left(\mathbf{p}_{\rho_{j}\left(l_{j}\right)}+\mathrm{R}_{3}^{-1}(\psi) \mathbf{p}_{l_{j}}\right)\right) \\
& \times\left(\mathscr{I}_{\boldsymbol{\rho}}^{\left\{N_{j}\right\}}\left(I, I_{3}\right) \prod_{j=1}^{K} \prod_{l_{j}=1}^{L_{j}} \delta_{\alpha_{\rho_{j}\left(l_{j}\right)} \alpha_{l_{j}}}+C \chi_{C} \overline{\mathscr{I}}_{\boldsymbol{\rho}}^{\left\{N_{j}\right\}}\left(I, I_{3}\right) \prod_{j=1}^{K} \prod_{l_{j}=1}^{L_{j}} \delta_{-\alpha_{\rho_{j}\left(l_{j}\right)} \alpha_{l_{j}}}\right)
\end{aligned}
$$

where the factors:

$$
\begin{aligned}
& \mathscr{I}_{\boldsymbol{\rho}}^{\left\{N_{j}\right\}}\left(I, I_{3}\right) \equiv\left[\prod_{j=1}^{K}\left\langle I_{j},\left\{I_{3}^{l_{j}}\right\}\right|\right]\left|I, I_{3}\right\rangle\left\langle I, I_{3}\right|\left[\prod_{j=1}^{K}\left|\left\{I_{j}, I_{3}^{\rho_{j}\left(l_{j}\right)}\right\}\right\rangle\right] \\
& \bar{I}_{\boldsymbol{\rho}}^{\left\{N_{j}\right\}}\left(I, I_{3}\right) \equiv\left[\prod_{j=1}^{K}\left\langle I_{j},\left\{I_{3}^{l_{j}}\right\}\right|\right]\left|I, I_{3}\right\rangle\left\langle I, I_{3}\right|\left[\prod_{j=1}^{K}\left|I_{j},\left\{-I_{3}^{\rho_{j}\left(l_{j}\right)}\right\}\right\rangle\right]
\end{aligned}
$$

are isospin coefficients which can be calculated numerically as a sum of recoupling coefficients, and the Kroneker's $\delta$ factors in (3.13) $\delta_{-\mathbf{q}_{\rho_{j}\left(l_{j}\right)}}^{\mathbf{q}_{l_{j}}}$ and $\delta_{\mathbf{q}_{\rho_{j}\left(l_{j}\right)}}^{\mathbf{q}_{l_{j}}}$ come from vanishing scalar products between single particle states with different baryonic number and strangeness. Indeed, integrals over particle momenta in Eq. (3.10) cannot be solved analytically. The only attempt to evaluate a similar expression for a gas of spinless particles in the large volume limit was made in [18] by using a Monte-Carlo method. We used a similar numerical method for the calculation of Eq. (3.10) which is described in [16].

\footnotetext{
${ }^{3}$ This factor includes additional charge conjugation phase factors of light-flavoured mesons.
} 


\section{Interacting hadron gas}

In this work we will account for interactions treating on an equal footing (as free particles) resonances and stable hadrons with respect to strong interactions, by using the definition (3.4) for the probability. This approach, known as the hadron resonance gas model, is quite popular and well known because matches the physical intuition (in the zero width limit, it is a natural expectation that resonances behave like stable particles), whereas it is not so for its theoretical basis which has been given by Dashen, Ma and Bernstein in the late 60's in ref. [19] and it is known as the Dashen Ma Bernstein theorem (DMB). Particularly, the authors wrote the microcanonical partition function in a very convenient way as the sum of the free microcanonical partition function plus an interaction term involving the scattering matrix. A more detailed discussion of this theorem is postponed to a forthcoming publication [20], nevertheless, it is worth to stress in advance some important aspects. First of all, it must be mentioned that the DMB theorem (thus the hadron resonance gas model) is valid in the infinite-volume limit. In fact, a derivation of the theorem for a finite volume seems still to be unpractical, therefore, one should keep in mind that, in what follows, finite-volume corrections of the interactions contribution are neglected. Secondly, in the simple hadron resonance gas model (as it will be shown in [20]) part of the interaction term is not included as it depend on unknown coefficients and also interference terms between nearby overlapping resonances are neglected.

Unfortunately, a precise quantitative estimation of these approximations on the production rate of exclusive channels is still an open issue. However, there are arguments (whose discussion will be in [20]) to argue that our final results shouldn't be dramatically affected by them. This is also supported by the success of the hadron resonance gas model in accounting for interactions among hadrons in high energy collisions, where many quantitative analyses have been made [1,2].

In order to get the microcanonical weight of a final channel with only strongly stable hadrons one should then make a weighted sum of free microcanonical weights (3.13) of channels including both hadrons and resonances (handled as free particles with distributed mass) eventually decaying into the final hadrons of the channel.

Therefore, given a final channel $\left\{N_{j}\right\}$, one should find all possibles parent channels whose hadrons and resonances decay into it. The search for all parent channels is a multi-step recursive problem in that many generations can be involved. If we denote by $\left\{N_{j}\right\}_{(1)}$ a channel which can directly decay into the channel $\left\{N_{j}\right\}$, by $\left\{N_{j}\right\}_{(2)}$ a channel which can directly decay in $\left\{N_{j}\right\}_{(1)}$ and so on, one has to find all possible decay trees like those shown in Fig. (2). In view of the large number of resonances, this task is not a trivial one; a suitable algorithm has been devised which is described in [16]. The probability $\rho_{\left\{N_{j}\right\}}$ of observing a final channel $\left\{N_{j}\right\}$ can then be expressed as a finite sum:

$$
\begin{aligned}
\rho_{\left\{N_{j}\right\}} & \propto \omega_{\left\{N_{j}\right\}} \equiv \Omega_{\left\{N_{j}\right\}}+\mathrm{BR}_{(1)} \Omega_{\left\{N_{j}\right\}_{(1)}}+\mathrm{BR}_{(2)} \mathrm{BR}_{(1)} \Omega_{\left\{N_{j}\right\}_{(2)}}+\ldots \\
& +\mathrm{BR}_{(1)^{\prime}} \Omega_{\left\{N_{j}\right\}_{(1)^{\prime}}}+\mathrm{BR}_{(2)^{\prime}} \mathrm{BR}_{(1)^{\prime}} \Omega_{\left\{N_{j}\right\}_{(2)^{\prime}}}+\ldots
\end{aligned}
$$

where $\mathrm{BR}_{(i)}$ is the product of branching ratios of particles in the channel $\left\{N_{j}\right\}_{(i)}$ decaying into particles in the channel $\left\{N_{j}\right\}_{(i-1)}$ and where $\omega_{\left\{N_{j}\right\}}$ is the channel weight where contributions of parent channels are included. 

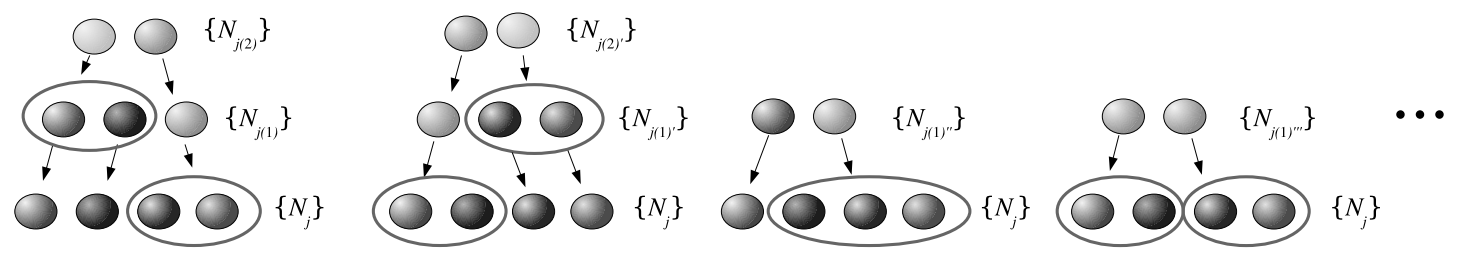

Figure 2: Examples of possible decay trees for a four particles channel. Circles encompass decay products of the particle at higher level.

\subsection{Strangeness suppression factor}

We allow deviations from statistical equilibrium of channels involving particles with strange valence quarks introducing a phenomenological parameter, the strangeness suppression factor $\gamma_{S}$. This parameter has been widely used in inclusive hadron multiplicity analyses and it is likely to be needed also in the analysis of exclusive rates at low energy. In order to have agreement with the $\gamma_{S}$ definition in the canonical and grand-canonical limit, one should multiply the microcanonical weight of a channel by $\gamma_{S}^{s_{j}} s_{j}$ being the number of valence strange quarks of each particle:

$$
\Omega_{\left\{N_{j}\right\}} \rightarrow\left[\prod_{j=1}^{k}\left(\gamma_{S}^{s_{j}}\right)^{N_{j}}\right] \Omega_{\left\{N_{j}\right\}}
$$

The $\gamma_{S}$ factor also applies to neutral mesons with valence strange quarks like $\eta, \phi$ etc. Since the wavefunction of such particles is in general a superposition like $C_{\mathrm{u}} \mathrm{u} \overline{\mathrm{u}}+C_{\mathrm{d}} \mathrm{d} \overline{\mathrm{d}}+C_{\mathrm{s}} \mathrm{s} \overline{\mathrm{s}}$ with $\left|C_{\mathrm{u}}\right|^{2}+$ $\left|C_{\mathrm{d}}\right|^{2}+\left|C_{\mathrm{s}}\right|^{2}=1$, only the component $\mathrm{s} \overline{\mathrm{s}}$ of the wavefunction is suppressed, i.e. we multiply by:

$$
\left|C_{\mathrm{S}}\right|^{2} \gamma_{S}^{2}+\left(1-\left|C_{\mathrm{s}}\right|^{2}\right)
$$

We have used mixing angles quoted by the Particle Data Book [21].

\section{Antiproton-proton annihilation at rest}

In the past, there have been several attempts to reproduce hadron multiplicities and some multi-pion(kaon) channel production ratios in low energy $\mathrm{e}^{+} \mathrm{e}^{-}$collisions [22] and $\mathrm{p} \overline{\mathrm{p}}$ annihilation at rest [10] by using statistical-thermodynamical or statistical-inspired models. Yet, to simplify numerical calculations, none of them properly took into account the full set of conservation laws, which is a clear drawback because in few body decays all of them are indispensable. Nowadays, thanks to the increased computing power and to the purposely devised numerical techniques, we are in a position to make a thorough test of the statistical ansatz in a more rigorous formulation, taking into account properly conserved quantities and the finite volume of the hadron-emitting source.

Antiproton-proton annihilation at rest is the system where much data on exclusive decays channels in 2, 3 and 4 bodies have been collected. However, the annihilation proceeds from a mostly unknown mixture of angular momentum and isospin states and the quantitative analysis 
turns out to be quite complicated. Most of the data analyzed were taken by stopping low-energy antiprotons beams in liquid hydrogen. In this situation, a protonium atomic state is formed, and the annihilation takes place with the orbital angular momentum $l=0$ or $l=1$, whereas states with higher values of the angular momentum are strongly suppressed [23]. For $l=0$ the initial states are the spin-singlet ${ }^{1} S_{0}$ and the spin triplet ${ }^{3} S_{1}$ with quantum numbers:

$$
J^{P C}\left({ }^{1} S_{0}\right)=0^{-+} \quad \text { and } \quad J^{P C}\left({ }^{3} S_{1}\right)=1^{--}
$$

whereas for $l=1$ we have:

$$
J^{P C}\left({ }^{1} P_{1}\right)=1^{+-}, \quad J^{P C}\left({ }^{3} P_{0}\right)=0^{++}, \quad J^{P C}\left({ }^{3} P_{1}\right)=1^{++}, \quad J^{P C}\left({ }^{3} P_{2}\right)=2^{++} .
$$

Altogether, there are two initial wavefunctions for annihilations occurring in $S$-wave and four wave functions for annihilations occurring in $P$-wave. The precise fraction of $P$-wave annihilations in liquid (that we will denote with $f_{P}$ ) has been the subject of a longstanding controversy as it was measured by several groups with inconsistent results [23]. >From the latest data coming from the Crystal Barrel experiment, the value one obtains is $f_{P}=(13 \pm 4) \%$ [23].

In order to write the branching ratio of a certain final channel $c h$ simplifying the notations as much as possible, let us label with 1 and 2 the states in (5.1) so that $J^{P C}(1)=0^{-+}$with $J_{1}=0$ and $J^{P C}(2)=1^{--}$with $J_{2}=1$. Accordingly, the states in (5.2) will be labeled with $3,4,5,6$ respectively. Altogether, if we let $\mathrm{BR}_{i}(c h)$ be the branching ratio in the final channel $c h$ of a given initial state $i$, the total branching ratio tuns out to be:

$$
\mathrm{BR}(c h)=\left(1-f_{P}\right) \sum_{i=1}^{i=2} w_{i}^{S} e_{i} \mathrm{BR}_{i}(c h)+f_{P} \sum_{i=3}^{i=6} w_{i}^{P} e_{i} \mathrm{BR}_{i}(c h)
$$

where the first and the second sum account respectively for $S$-wave and $P$-wave annihilations. The symbols $w_{i}^{S}$ and $w_{i}^{P}$ in (5.3) stand for the purely statistical weights of the various initial wavefuntions, namely:

$$
w_{i}^{S}=\frac{2 J_{i}+1}{4}, \quad w_{i}^{P}=\frac{2 J_{i}+1}{12},
$$

whereas the factors $e_{i}$ are called enhancement factors and describe the departure from a pure statistical population $\left(e_{i}=1\right)$. This phenomenon is due to the strong Stark mixing which constantly repopulates the fine and hyperfine levels in liquid hydrogen [23]. These factors have been calculated in ref. [24] with an x-ray cascade calculation by using three different optical potential models of the p $\bar{p}$ interaction: DR1, DR2 [25] and KW [26]. In tab. 1 the values obtained therein are reported.

The eq. (5.3) takes into account all possible annihilation spin states. Yet, also total isospin can assume two values: $I=0$ and $I=1$. Therefore, we will assume an unknown statistical mixture of $I=0$ and $I=1$ initial state disregarding interference terms and introducing a free parameter $f_{I=0}$, i.e. the fraction of $I=0$ state:

$$
f_{I=0}|0,0\rangle\left\langle 0,0\left|+\left(1-f_{I=0}\right)\right| 1,0\right\rangle\langle 1,0|
$$

where isospin states have been denoted has $\left|I, I_{3}\right\rangle$. 


\begin{tabular}{|c|c|c|c|}
\hline & DR1 & DR2 & KW \\
\hline \hline$e_{1}$ & 1.032 & 1.028 & 1.060 \\
\hline$e_{2}$ & 0.989 & 0.991 & 0.980 \\
\hline$e_{3}$ & 0.856 & 0.933 & 0.809 \\
\hline$e_{4}$ & 2.556 & 2.076 & 2.176 \\
\hline$e_{5}$ & 0.685 & 0.541 & 0.703 \\
\hline$e_{6}$ & 0.964 & 1.041 & 1.058 \\
\hline
\end{tabular}

Table 1: Enhancement factors obtained with an $\mathrm{x}$-ray cascade calculation by using three different optical potential models: DR1, DR2 [25] and KW [26]

\begin{tabular}{|c|c|c|c|c|c|}
\hline$f_{I=0}^{1}$ & $f_{I=0}^{2}$ & $f_{I=0}^{3}$ & $f_{I=0}^{4}$ & $f_{I=0}^{5}$ & $f_{I=0}^{6}$ \\
\hline 0.56 & 0.56 & 0.62 & 0.95 & 0.13 & 0.60 \\
\hline
\end{tabular}

Table 2: Isospin probabilities for $\mathrm{p} \overline{\mathrm{p}}$ atomic states.

Therefore, the full expression of the branching ratio turns out to be:

$$
\begin{aligned}
\mathrm{BR}(c h) & =\left(1-f_{P}\right) \sum_{i=1}^{i=2} w_{i}^{S} e_{i}\left[f_{I=0}^{i} \mathrm{BR}_{i}^{I=0}(c h)+\left(1-f_{I=0}^{i}\right) \mathrm{BR}_{i}^{I=1}(c h)\right] \\
& +f_{P} \sum_{i=3}^{i=6} w_{i}^{P} e_{i}\left[f_{I=0}^{i} \mathrm{BR}_{i}^{I=0}(c h)+\left(1-f_{I=0}^{i}\right) \mathrm{BR}_{i}^{I=1}(c h)\right]
\end{aligned}
$$

where $\mathrm{BR}_{i}^{I=0}(c h)$ and $\mathrm{BR}_{i}^{I=1}(c h)$ are the branching ratio in the final channel $c h$ when the spin state is $i$ and the isospin state is respectively $I=0$ and $I=1$. The factor $f_{I=0}^{i}$ is the probability that the state $i$ has isospin 0 and, in principle, it can vary from 0 to 1 . For a pure $\mathrm{p} \overline{\mathrm{p}}$ initial state we have $f_{I=0}^{i}=1 / 2$ for any $i$. Nevertheless, at short distances $(r \lesssim 1 \mathrm{fm})$, the neutron-antineutron admixture in the protonium wave function can dramatically affect the sharing of the $I=0$ and $I=1$ states within a given $J^{P C}$ state. These probabilities have been calculated in [27] by introducing mesonexchange potentials to describe the $\mathrm{p} \overline{\mathrm{p}}$ interaction. Their values reported in tab. 2 refers to DR2 optical model with $2 \pi+\omega$ exchange potential.

In order to calculate the branching ratio in eq. (5.5) with SHM, one need the branching ratios in each possible $J^{P C}$ and isospin state. In other words, for a given final channel $\left\{N_{j}\right\}$, we must evaluate:

$$
\operatorname{BR}_{i}^{I}\left(\left\{N_{j}\right\}\right) \equiv \rho_{\left\{N_{j}\right\}}^{I, i}=\frac{\omega_{\left\{N_{j}\right\}}^{I, i}}{\Omega_{I, i}} \quad I=0,1 ; \quad i=1, \ldots, 6
$$

where $\rho_{\left\{N_{j}\right\}}^{I, i}$ and $\omega_{\left\{N_{j}\right\}}^{I, i}$ are the probability and the channel weight defined in (4.1), and $\Omega_{I, i}$ is the microcanonical partition function, which is obtained as a sum over all possible channels according to eq. (3.7). In eq. (5.6), $I$ stands for the isospin $(I=0$ or $I=1)$ and the index $i$ labels the six possible $J^{P C}$ states in (5.1) and (5.2).

As a first analysis, we made a preliminary comparison with a set of two-mesons exclusive channels. In this case the channel weight $\omega_{\left\{N_{j}\right\}}^{I, i}$ coincide with $\Omega_{\left\{N_{j}\right\}}^{I, i}$ (see eq.(4.1)). The result we 
obtained is shown in fig. 3. This plot has been obtained performing a coarse scan of the parameters

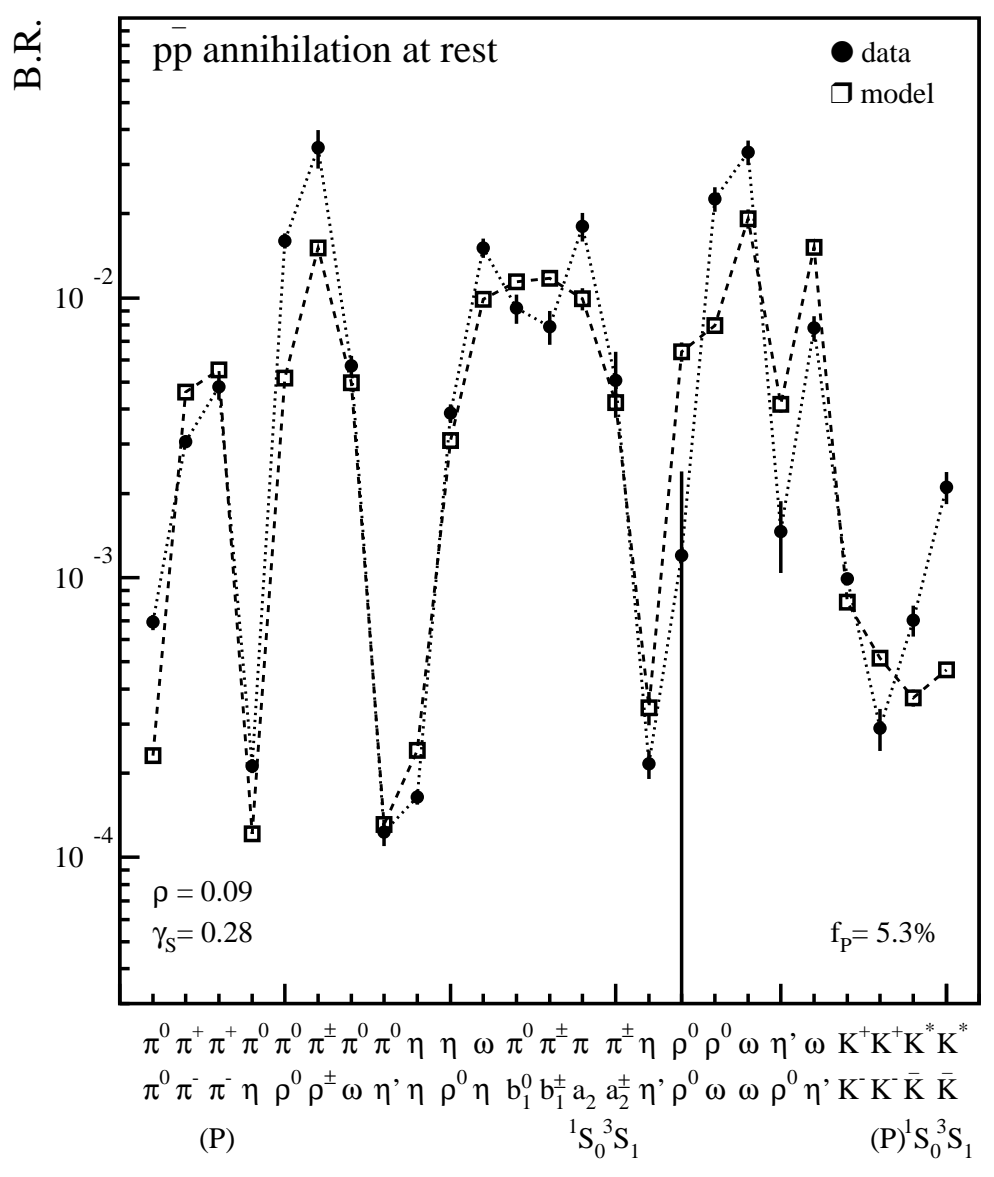

Figure 3: Comparison between measured branching ratios (black dots) of various two-mesons channels and the prediction of the SHM (white squares). We used experimental data quoted in ref. [23]. Branching ratios (where not differently specified) refer to measures made in liquid hydrogen without any initial-state selection. Conversely, when the symbols ${ }^{1} S_{0},{ }^{3} S_{1}$ and $(P)$ appear, only annihilations from ${ }^{1} S_{0},{ }^{3} S_{1}$ or $P$-wave initial state have been selected.

$\gamma_{s}$ and the energy density $\rho$ defined as:

$$
\rho \equiv \frac{M}{V}=\frac{3 M}{4 \pi R^{3}}
$$

where $M \equiv \sqrt{s}$ is the cluster's mass. We allowed the parameters $e_{i}$ to vary between the minimum and the maximum value predicted by the different optical potentials (see tab. 1) enforcing the normalizing conditions:

$$
\sum_{i=1}^{i=2} e_{i} w_{i}^{S}=1, \quad \sum_{i=3}^{i=6} e_{i} w_{i}^{P}=1 .
$$

The parameters $f_{I=0}^{i}$ have been also allowed to vary within reasonable limits given by the minimum and maximum values in tab. 3; whereas for $f_{P}$ we have chosen the range $0.5 \% \leq f_{P} \leq 17 \%$ : The 


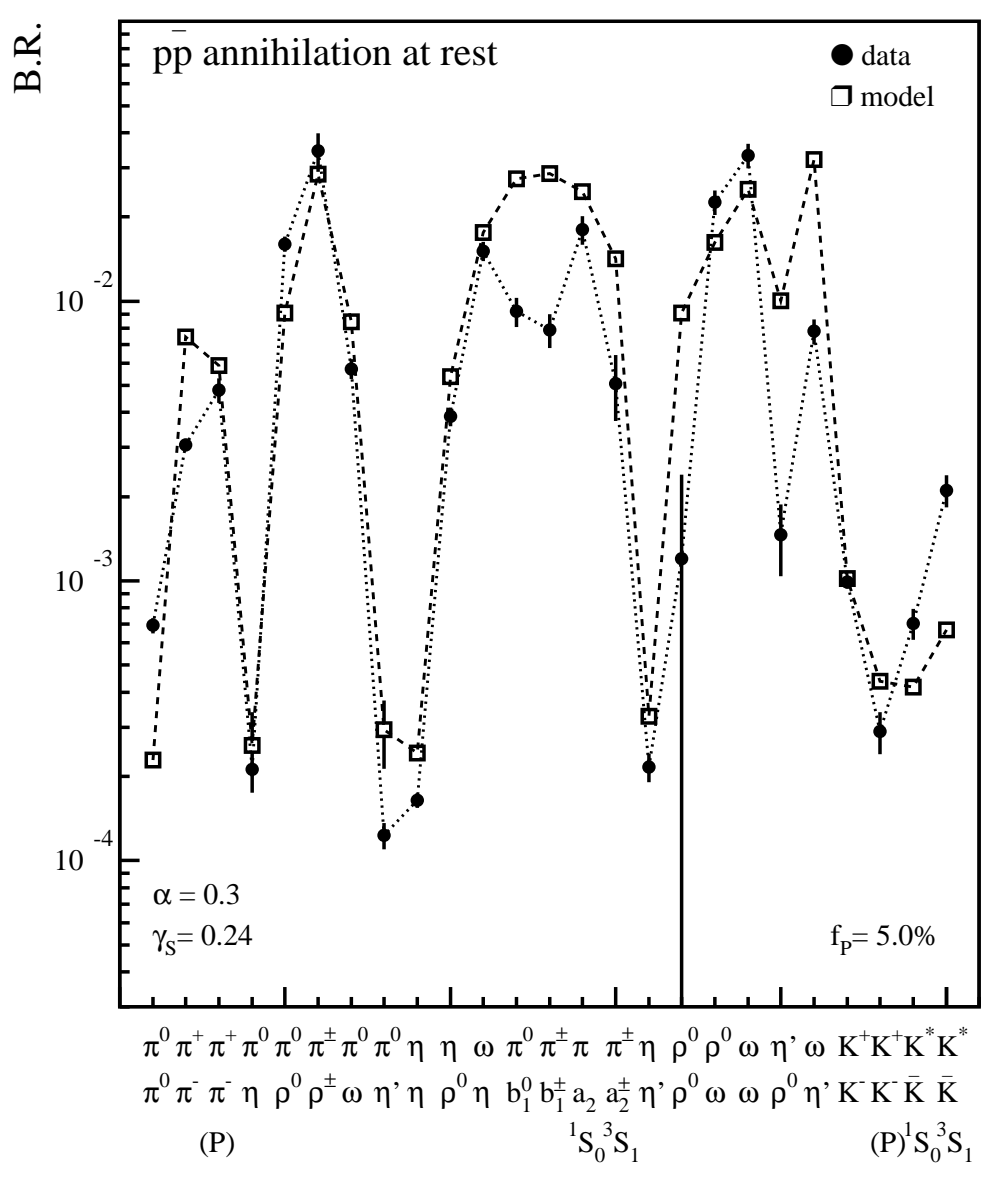

Figure 4: Comparison between measured branching ratios (black dots) of various two-mesons channels and the prediction of the Phase space dominance model (white squares). We used experimental data quoted in ref. [23]. Branching ratios (where not differently specified) refer to measures made in liquid hydrogen without any initial-state selection. Conversely, when the symbols ${ }^{1} S_{0},{ }^{3} S_{1}$ and $(P)$ appear, only annihilations from ${ }^{1} S_{0},{ }^{3} S_{1}$ or $P$-wave initial state have been selected.

\begin{tabular}{|c|c|c|c|c|c|c|}
\hline & $f_{I=0}^{1}$ & $f_{I=0}^{2}$ & $f_{I=0}^{3}$ & $f_{I=0}^{4}$ & $f_{I=0}^{5}$ & $f_{I=0}^{6}$ \\
\hline $\min$ & 0.5 & 0.5 & 0.6 & 0.9 & 0.05 & 0.5 \\
\hline $\max$ & 0.6 & 0.6 & 0.7 & 1.0 & 0.2 & 0.7 \\
\hline
\end{tabular}

Table 3: Maximum and minimum allowed values of the isospin probabilities.

best values of the whole set parameters as been chosen as the set that minimize the function $\chi^{2}$ defined as:

$$
\chi^{2}=\sum_{c h} \frac{\left(\mathrm{BR}^{\exp .}(c h)-\mathrm{BR}^{\mathrm{SHM}}(c h)\right)^{2}}{\sigma^{\exp \cdot(c h)^{2}+\sigma^{\mathrm{SHM}}(c h)^{2}}}
$$

where the sum runs over all measured two-mesons channels. In eq. (5.9), 
$\sigma^{\text {exp. }}(c h)$ and $\sigma^{\mathrm{SHM}}($ ch $)$ are the uncertainties on measured branching ratios and on the model calculation respectively. The latter being nothing but the statistical error owing to the finite number of sampling in Monte-Carlo integration. The same analysis has been repeated by assuming the phase space dominance model (fig. 4). As one can see, in both cases, the order of magnitude of exclusive ratios is fairly reproduced although some channel shows significant discrepancies. This might owe to a too simple description of interactions among stable hadrons (with the hadronresonance gas model), or to neglected effects like isospin interference. Indeed, also the very fact that proton and antiproton are particles endowed by inner structure could play an important role. This argument is also supported by ongoing analyses of SHM on $\mathrm{e}^{+} \mathrm{e}^{-}$collisions at $\sqrt{s} \lesssim 2.5 \mathrm{GeV}$ (that will be shown in a forthcoming paper [17]) that seems to show a good agreement with experimental data. However, if from one side this result seems to be encouraging as both models catch a large part of the phenomenology, on the other side this test cannot help us to distinguish between phase space dominance and SHM. In fact, though a closer look reveals that the SHM calculation is slightly closer to the data, both models give too similar results and no clearcut conclusions can be drawn.

\section{Conclusions}

We have performed a preliminary test of the SHM on production rates of exclusive channels in $\mathrm{p} \overline{\mathrm{p}}$ annihilation at rest. The calculation of such quantities required a formulation of the SHM in its fundamental microcanonical framework, enforcing the maximal set of conservation laws relevant to strong interaction and space-time symmetries according to the formalism developed in refs. $[11,12]$. The complication of the annihilation process, which proceeds from many initial $p \bar{p}$ atomic bound states, required the introduction of several free parameters, which made the test not as clean as it would have been desirable. Altogether, the SHM fairly reproduces the general trend of the experimental data although large discrepancies appear for some channels. The analysis has been repeated by assuming the phase space dominance model, that is the main option (besides SHM) to account for the apparent thermal-like features of the hadronization process. The result we obtained is qualitatively the same as SHM and, unfortunately, this specific test proved to be unfit to discriminate between these two different scenarios.

\section{References}

[1] F. Becattini, Z. Phys. C 69 (1996) 485;

F. Becattini, Proc. of XXXIII Eloisatron Workshop on "Universality Features in Multihadron Production and the leading effect" (1996) 74, hep-ph 9701275; F. Becattini, U. Heinz, Z. Phys. C 76 (1997) 269;

F. Becattini, G. Passaleva, Eur. Phys. J. C 23 (2002) 551.

[2] P. Braun-Munzinger, J. Stachel, J. P. Wessels and N. Xu, Phys. Lett. B 365 (1996) 1;

J. Cleymans, D. Elliott, H. Satz and R. L. Thews, Z. Phys. C 74 (1997) 319;

F. Becattini, J. Manninen and M. Gazdzicki, Phys. Rev. C 73 (2006) 044905;

W. Broniowski, A. Baran and W. Florkowski, Acta Phys. Polon. B 33 (2002) 4235.

[3] F. Becattini, U. Heinz, Z.Phys C 76, (1997) 269;

H. Satz, Nucl. Phys. Proc. Suppl. 94, (2001) 204; 
R. Stock, Phys. Lett. B 456, (1999) 277;

Y. Dokshitzer, Acta Phys. Polon. B 36 (2005) 361;

A. Bialas, Phys. Lett. B 466 (1999) 301;

V. Koch, Nucl. Phys. A 715 (2003) 108;

L. McLerran, arXiv:hep-ph/0311028;

[4] D. Kharzeev, Nucl. Phys. A 774 (2006) 315.

[5] J. Hormuzdiar,S. D. H. Hsu, G. Mahlon Int. J. Mod. Phys. E 12 (2003) 649.

[6] U. Heinz, Nucl. Phys. A 661 (1999) 140.

[7] F. Becattini, J. Phys. Conf. Ser. 5 (2005) 175.

[8] R. Hagedorn, N. Cim. Suppl. 3 (1965) 147.

[9] D. Kharzeev, Eur. Phys. J. A 29 (2006) 83;

H. Satz, PoS C POD2006 (2006) 039 [arXiv:hep-ph/0612151];

P. Castorina, D. Kharzeev and H. Satz, Eur. Phys. J. C 52 (2007) 187.

[10] W. Blumel, P. Koch, U. Heinz Z. Phys. C 63 (1994) 637.

[11] F. Becattini, L. Ferroni, Eur. Phys. J. C 51 (2007) 899.

[12] F. Becattini, L. Ferroni, arXiv:0707.0793 [nucl-th].

[13] F. Becattini, L. Ferroni, Eur. Phys. J. C 35 (2004) 243.

[14] F. Becattini, L. Ferroni, Eur. Phys. J. C 38 (2004) 225.

[15] F. M. Liu, K. Werner, J. Aichelin Phys. Rev. C 68 (2003) 024905.

[16] L. Ferroni, "The microcanonical ensemble of the relativistic hadron gas". PhD thesis, University of Florence (2006).

[17] F. Becattini, L. Ferroni, in preparation.

[18] F. Cerulus, N. Cim. X22, (1961) 958-995.

[19] R. Dashen, S. Ma, H. Bernstein, Phys. Rev. 187 (1969) 345;

R. Dashen, R. Rajamaran, Phys. Rev. D 10 (1974) 694.

[20] F. Becattini, in preparation.

[21] W. M. Yao et al. [Particle Data Group], J. Phys. G 33 (2006) 1.

[22] G. Eilam, C. Hojvat, B. Margolis, W. J. Meggs, S. C. Frautschi and M. Pripstein, Lett. Nuovo Cim. 14 (1975) 108 ;

W. Ochs, MPI-PAE/Exp El-107, Invited talk given at Europhysics Study Conf. on Jet Structure from Quark and Lepton Interactions, Erice, Italy, Sep 12-17, 1982;

C. E. Carlson and F. Gross, Phys. Rev. D 14 (1976) 1858.

[23] C. Amsler, Rev. Mod. Phys. 70 (1998) 1293, and references therein.

[24] C. J. Batty, Nucl. Phys. A 601 (1996) 425.

[25] J. M. Richard, M. E. Sainio, Phys. Lett. B 110 (1982) 349;

C. B. Dover, J. M. Richard, Phys. Rev. C 21 (1980) 1466.

[26] M. Kono, W. Weise, Nucl. Phys. A 454 (1986) 429.

[27] C. B. Dover, J. M. Richard, J. Carbonell, Phys. Rev. C 44 (1991) 1281-1288. 